\title{
Lattice Signatures without Trapdoors
}

\author{
Vadim Lyubashevsky* \\ INRIA / École Normale Supérieure
}

\begin{abstract}
We provide an alternative method for constructing latticebased digital signatures which does not use the "hash-and-sign" methodology of Gentry, Peikert, and Vaikuntanathan (STOC 2008). Our resulting signature scheme is secure, in the random oracle model, based on the worst-case hardness of the $\tilde{O}\left(n^{1.5}\right)$-SIVP problem in general lattices. The secret key, public key, and the signature size of our scheme are smaller than in all previous instantiations of the hash-and-sign signature, and our signing algorithm is also quite simple, requiring just a few matrix-vector multiplications and rejection samplings. We then also show that by slightly changing the parameters, one can get even more efficient signatures that are based on the hardness of the Learning With Errors problem. Our construction naturally transfers to the ring setting, where the size of the public and secret keys can be significantly shrunk, which results in the most practical to-date provably secure signature scheme based on lattices.
\end{abstract}

\section{Introduction}

The versatility of lattice-based cryptography has elevated it to the status of a promising potential alternative to cryptography based on standard security assumptions such as factoring and discrete log. But before lattices can become a viable replacement for number-theoretic schemes, it is crucial to have efficient lattice-based constructions of the most ubiquitous cryptographic primitives in practical applications, which are arguably encryption schemes and digital signatures.

On the encryption front, lattice-based schemes have been making a lot of progress with recent provably-secure schemes [39|28|24|41] being almost as practical as (and actually looking quite similar to) the deployed NTRU [20] encryption scheme, which in turn has many advantages over number theory-based schemes. Lattice-based signatures, on the other hand, have been a different story. An early attempt at lattice-based signatures was the GGH scheme [18] but it was almost immediately shown to be weaker than expected [33, and eventually completely broken [34. The NTRU signature scheme had an even more more tumultuous history since its introduction in 2001 [21], with attacks [17] being followed by fixes [19, until its basic version was also completely broken by Nguyen and Regev [34].

\footnotetext{
^ Work supported in part by the European Research Council.
} 
Provably secure lattice-based signature schemes were finally constructed in 2008, when Gentry, Peikert, and Vaikuntanathan [16] constructed a "hash-andsign" signature scheme based on the hardness of worst-case lattice problems and Lyubashevsky and Micciancio [27] constructed a one-time signature based on the hardness of worst-case ideal lattice problems. The hash-and-sign signatures were rather inefficient (with signatures being megabytes long) and the one-time signature, while being relatively short, still required Merkle trees to become a full-fledged signature. Building on [27, Lyubashevsky proposed a digital signature, using the Fiat-Shamir framework [12] based on the hardness of ideal lattice problems 26. This latter scheme has signature lengths on the order of 60000 bits for reasonable security parameters, and while closer to being practical, it is still not as small as one would like. Subsequently, lattice-based signature schemes without random oracles were also constructed [118, but they are all much less efficient in practice than their random oracle-using counterparts.

\subsection{Related Work and Our Results}

A common thread running through constructions of digital signatures in the random oracle model, whether using the hash-and-sign or the Fiat-Shamir technique [12, is to force the distribution of the signature to be statistically independent of the secret key. If this property is achieved, then by programming the random oracle, one can hope to produce the valid signatures requested by the potential forger in the security reduction, without knowing the secret key. Then, when the forger produces a signature of a new message, it can be used to solve the underlying hard problem. In the case of lattices, the underlying hard problem is usually the Small Integer Solution (SIS) problem in which one is given a matrix $\mathbf{A}$ and is asked to find a small vector $\mathbf{v}$ such that $\mathbf{A v}=0 \bmod q$. The length of $\mathbf{v}$ is very close to the length of signatures in the scheme, and thus the challenge for improving lattice-based signatures based on SIS is to reduce the norm of the signatures produced by the signing algorithm.

In lattice-based hash-and-sign signatures [16, every signer has a personal uniformly random public matrix $\mathbf{A} \in \mathbb{Z}_{q}^{n \times m}$ and an associated secret "trapdoor" $\mathbf{S} \in \mathbb{Z}_{q}^{m \times m}$ with small coefficients such that $\mathbf{A S}=0 \bmod q$. To sign a message $\mu$, the signer uses his secret key $\mathbf{S}$ to produce a short signature vector $\mathbf{z}$, whose distribution is independent of $\mathbf{S}$, such that $\mathbf{A z}=\mathrm{H}(\mu) \bmod q$, where $\mathrm{H}$ is a cryptographic hash function. Since the length of $\mathbf{z}$ roughly depends on the norms of the columns of $\mathbf{S}$, improving the hash-and-sign signature scheme involves coming up with better algorithms for generating the pairs $(\mathbf{A}, \mathbf{S})$ such that $\mathbf{S}$ has smaller dimensions and smaller coefficients. Using the original algorithm due to Ajtai [1], the signature scheme of [16] produced signatures of norm $\tilde{O}\left(n^{1.5}\right)$. A subsequent improvement of the key-generation algorithm by Alwen and Peikert [3] lowered the signature length to $\tilde{O}(n)$, and the very recent algorithm of Micciancio and Peikert 30] further reduces the constants (and removes some logarithmic factors) from the previous algorithms. 
There has been much less progress in the direction of building lattice-based signature schemes using the Fiat-Shamir technique. In fact, the only such scheme 1 is the ring-based one of Lyubashevsky 26, in which the signature vectors are of norm $\tilde{O}\left(n^{1.5}\right)$. The first contribution of this current work is adapting the ringSIS based scheme from [26] to one based on the hardness of the regular SIS problem which results in signatures of the same $\tilde{O}\left(n^{1.5}\right)$ length 2 . Our second contribution is analogous to what the works 33630 did for hash-and-sign signatures - reduce the signature length to $\tilde{O}(n)$ (of course the issues that have to be dealt with are completely different). Our third contribution is showing that the parameters of our scheme can be set so that the resulting scheme produces much shorter signatures, but is now based on the hardness of the Learning With Errors (LWE) problem 39 or on the hardness of a low-density version of the SIS problem. All our results very naturally carry over to the ring setting, where the key bit-size is reduced by a factor of approximately $n$ (some sample parameters are given in Figure 2).

Our signature scheme is also quite simple, requiring no pre-image sampling over arbitrary lattices. All we do is sample the Normal distribution over $\mathbb{Z}^{m}$, compute a vector-matrix product, do a random oracle query, compute another vector-matrix product (this time the vector is sparse), and rejection sample. In fact, in an online/offline setting where we can do pre-computations before being given the message to sign, the online phase simply consists of doing a few vector additions (since the matrix is being multiplied by a sparse vector) and rejection sampling.

\subsection{Techniques}

We now briefly sketch our signature scheme and describe the issues involved in lowering the size of the signature. The secret key is a matrix $\mathbf{S} \in \mathbb{Z}_{q}^{m \times k}$ with small coefficients, and the public key consists of the matrices $\mathbf{A} \in \mathbb{Z}_{q}^{n \times m}$ and $\mathbf{T}=\mathbf{A S} \bmod q$. The matrix $\mathbf{A}$ can be shared among all users, but the matrix $\mathbf{T}$ is individual. To sign a message, the signer first picks a vector $\mathbf{y} \in \mathbb{Z}_{q}^{m}$ according to some distribution $D$. Then he computes $\mathbf{c} \in \mathbb{Z}_{q}^{k}$ where $\mathbf{c} \leftarrow \mathrm{H}(\mathbf{A y} \bmod q, \mu)$, and computes the potential signature vector $\mathbf{z}=\mathbf{S c}+\mathbf{y}$ (there is no reduction modulo $q$ in this step). The vector $\mathbf{z}$, along with $\mathbf{c}$, will then be output as the signature based on some criteria with the end goal being that the distribution of $(\mathbf{z}, \mathbf{c})$ should be independent of the secret key matrix $\mathbf{S}$.

${ }^{1}$ We mention that the lattice-based identification schemes of Lyubashevsky [25] and Kawachi et al. 23], while may be converted into signature schemes, are inherently inefficient because every round of the ID scheme has soundness error at least $1 / 2$.

${ }^{2}$ As a side note to this first result, we think that it is interesting to point out that the ring-structure, which seemed so native to [26] (and to [27]), turns out to not actually provide any additional functionality, with its purpose being only to shorten the key-sizes and make operations more efficient. This somewhat resembles the recent developments in constructions of fully-homomorphic encryption schemes, where the additional structure of ideal lattices was crucially used in earlier constructions [14 1510, but was subsequently shown to be unnecessary 92 . 
Choosing when to output the pair $(\mathbf{z}, \mathbf{c})$ can be seen as a kind of rejection sampling. If $f$ and $g$ are probability distributions and $M \in \mathbb{R}$ is such that for all $x, f(x) \leq M g(x)$, then if one samples elements $z$ from $g$ and outputs them with probability $f(z) /(M g(z))$, the resulting distribution is exactly $f$, and the expected amount of time needed to output a sample is $M$.

Our goal, in the signature scheme above, is to come up with distributions $f$ and $D$ so that for all $\mathbf{x}$, two properties are satisfied: there is a small constant $M$ such that $f(\mathbf{x}) \leq M g(\mathbf{x})$, where $g$ is the distribution generated by first picking $\mathbf{y}$ from $D$ and adding it to $\mathbf{S c}$ for some random $\mathbf{c}$; and the expected value of vectors distributed according to $f$ (which is the length of the signature) is as small as possible. The idea in [26], when put into the above framework, was to choose $\mathbf{y}$ uniformly from an $m$-dimensional sphere $3 \beta_{r+v}$ of radius $r+v$, where $r$ is some number and $v$ is the maximum possible length of the vector Sc, and only output $\mathbf{z}$ if it fell into a sphere $\beta_{r}$ of radius $r$. It's not hard to check that if $f$ is the uniform distribution over the sphere $\beta_{r}$, then by setting $M=\operatorname{vol}\left(\beta_{r+v} / \beta_{r}\right) \approx(1+v / r)^{m}$, the distribution of $\mathbf{z}$ is exactly $f$. But in order to keep $M$ small, we need $r>m v=\tilde{\Theta}\left(m^{1.5}\right)=\tilde{\Theta}\left(n^{1.5}\right)$, and so the vectors $\mathbf{z}$ have length $\tilde{O}\left(n^{1.5}\right)$.

In our present work we show that we can do better by choosing $f$ and $D$ to be the $m$-dimensional Normal distribution with standard deviation $\sigma=\tilde{\Theta}(v)=$ $\tilde{\Theta}(\sqrt{m})$, and only require that $f(\mathbf{x}) \leq M g(\mathbf{x})$ for the $\mathbf{x}$ that are not too big. We can then show that $M$ can be set to a constant, and the rejection sampling algorithm produces a distribution that is statistically close to the distribution of $f$. This means that the expected value of the length of the signature of $\mathbf{z}$ is $\sigma \sqrt{m}=\tilde{O}(m)=\tilde{O}(n)$. We prove the technical rejection sampling theorem in Section 3 and then prove the security of the above signature scheme based on the hardness of the SIS problem in Section 4 .

Notice that the length of the signature is greatly affected by the parameter $m$, and lowering $m$, while leaving everything else the same would produce even shorter signatures. The danger of doing this is that the problem of recovering $\mathbf{S}$ when given A and AS mod $q$ now becomes easier (and is no longer based on the SIS problem). The intuition is then to set all the parameters so that the hardness of recovering the secret key is equal, in practice, to the hardness of forging a signature. In Section 5 we explain how the parameters can be significantly lowered by making our scheme be based on the LWE problem instead of on SIS.

\subsection{A Practical Comparison with Hash-and-Sign Signatures}

On the theoretical side, both the scheme constructed in this paper and the hash-and-sign scheme that uses the trapdoor sampling algorithms of [3,36] are based on the hardness of finding a vector of length $\tilde{O}(n)$ in SIS instances, which by the worst-case to average-case reduction of Micciancio and Regev [31 is as hard as solving approximate SIVP with a factor of $\tilde{O}\left(n^{1.5}\right)$ in all $n$-dimensional

${ }^{3}$ In [26], it was actually a box, but it does not make a difference for the analysis here. 
lattices. On the practical side, however, the bit-length of our signature and keys (see Figure 2) are approximately two orders of magnitude smaller for the same security level (see 40 and also Figure 2 in 30]). This is mostly due to the constants that are hidden in the big-Oh notation of the trapdoor generation algorithms of [3] and 36].

As mentioned earlier, in a concurrent and independent work, Micciancio and Peikert greatly improved the constants, and in some cases even removed some logarithmic factors, in the trapdoor sampling algorithms 30. While the proof techniques are completely different, there are some high-level similarities between the two schemes. The public key in our scheme is (A, AS) where $\mathbf{A}$ is a random matrix $\bmod q$ and $\mathbf{S}$ is a secret matrix with small coefficients. In 30 , the public key is $(\mathbf{A}, \mathbf{A} \mathbf{S}+\mathbf{G})$ where $\mathbf{G}$ is an additional public matrix with a very "simple" form. In our scheme, the signature of a message is an ordered pair $(\mathbf{S c}+\mathbf{y}, \mathbf{c})$ where $\mathbf{c}$ is a function (that invokes a random oracle) of the message and the vector $\mathbf{y}$ is there to "hide" the shift $\mathbf{S c}$; while in 30 , the signature is $\left(\mathbf{S c}+\mathbf{y}_{1}, \mathbf{c}+\mathbf{y}_{2}\right)$ where $\mathbf{c}$ is a (different, random oracle-invoking) function of the message and $\mathbf{y}_{i}$ also serve the purpose of hiding the shift Sc (and $\mathbf{c}$ itself). While the schemes may look similar, under the surface they behave rather differently.

The most interesting and significant difference occurs in the way the signatures are generated. In our scheme, the vector $\mathbf{c}$ is a very sparse $-1 / 0 / 1$ vector whose entropy is as small as the security parameter, but we must output it as part of the signature. In 30, however, the size of the elements in c depends inversely on the number of columns of $\mathbf{S}$, but one only outputs a perturbed version of $\mathbf{c}$ as part of the signature. Notice that the size of our signature is therefore dominated by the number of rows of $\mathbf{S}$ multiplied by the number of bits needed to represent elements in the vector $\mathbf{S c}+\mathbf{y}$, whereas in [30], the number of columns of $\mathbf{S}$ may also play a significant role in the signature length.

The advantage in [30] due to the fact that $\mathbf{c}$ is never output in the clear is that they may tailor the perturbations $\mathbf{y}_{1}, \mathbf{y}_{2}$ to the particular $\mathbf{S}$ that they are supposed to hide, which allows these perturbations to be smaller than ours in the case that $\mathbf{S}$ has enough columns to allow c to be "small enough". When instantiating both signature schemes based on the worst-case hardness of the SIS problem, $\mathbf{S}$ needs to have a large number of rows, and thus the fact that the bit-size of the entries of the signature from [30] is smaller than of those in our scheme, may make the scheme from 30. more compact. On the other hand, if one is to instantiate the more practical version of the schemes based on the hardness of the LWE problem, then the number of rows in $\mathbf{S}$ could be significantly smaller, and thus the fact that the size of our signature does not depend on the number of columns of $\mathbf{S}$ gives it an advantage over the one in [30. We direct the reader to our sample instantiations in Figure 2 where one can see the signature size rapidly decreasing as the number of rows (denoted by $m$ ) shrinks. The trade-off is that as the number of rows shrinks, the worst-case hardness assumption becomes stronger, but it is still believed that the security of the average-case problem remains the same (see Section 2 and the full version of this work). 
Additionally, the number of columns in our secret key $\mathbf{S}$ needs to only be large enough to support multiplication by $\mathbf{c}$, which allows the number of columns to be significantly smaller than in the secret key of [30, where, for technical reasons, reducing the number of columns of $\mathbf{S}$ ends up increasing the coefficients of $\mathbf{c}$, and thus possibly increasing the size of the signature. This allows our secret key to be smaller that the one in 30 . Compared to the one concrete instantiation (based on the hardness of the SIS problem) provided in 30, where the key size is approximately $2^{26.5}$ bits and the signature is a 13800 dimensional vector of length 92000 , thus requiring at least $13800 \cdot \log (92000 / \sqrt{13800}) \approx 130000$ bits to represent, for the same security level, some of our instantiations have the signature bit-length about $25 \%$ longer, with the benefit of having the keys be about 10 times smaller (column I of Figure 2). For different instantiations, we can have the signature bit-length be about $45 \%$ shorter and have the same key size (column III of Figure 2).

\subsection{Notation}

Throughout the paper, we will assume that $q$ is a small (i.e. polynomial-size) prime number and elements in $\mathbb{Z}_{q}$ are represented by integers in the range $\left[-\frac{q-1}{2}, \frac{q-1}{2}\right]$. We will represent vectors by bold-face letters, and matrices by bold-face capital letters. We will assume that all vectors are column vectors, and $\mathbf{v}^{T}$ will denote the transpose of the vector $\mathbf{v}$. The $\ell_{p}$ norm of a vector $\mathbf{v}$ is denoted by $\|\mathbf{v}\|_{p}$, and we will usually avoid writing the $p$ for the $\ell_{2}$ norm. Whenever dealing with elements that are in $\mathbb{Z}_{q}$, we always explicitly assume that all operations in which they are involved end with a reduction modulo $q$. Thus for a matrix $\mathbf{A} \in \mathbb{Z}_{q}^{n \times n}$ and a vector $\mathbf{s} \in \mathbb{Z}^{n}$, the product $\mathbf{A s}$ is a vector in $\mathbb{Z}_{q}^{n}$. For a distribution $\mathcal{D}$, we use the notation $x \stackrel{\$}{\leftarrow}$ to mean that $x$ is chosen according to the distribution $\mathcal{D}$. If $S$ is a set, then $x \stackrel{\$}{\leftarrow} S$ means that $x$ is chosen uniformly at random from $S$. For an event $E$, we write $\operatorname{Pr}\left[E ; x_{1} \stackrel{\$}{\leftarrow} \mathcal{D}_{1}, \ldots, x_{k} \stackrel{\$}{\leftarrow} \mathcal{D}_{k}\right]$ to mean the probability that $E$ occurs when the $x_{i}$ are chosen from distributions $\mathcal{D}_{i}$. All logarithms are base 2 .

\section{The SIS Problem and Its Variants}

In this section, we will define the average-case problems upon whose security our signature schemes will be based. All these problems fall into the category of the Small Integer Solution (SIS) problem, which is essentially the knapsack problem over elements in $\mathbb{Z}_{q}^{n}$.

Definition $2.1\left(\ell_{2}-\mathrm{SIS}_{q, n, m, \beta}\right.$ problem). Given a random matrix $\mathbf{A} \stackrel{\$}{\leftarrow} \mathbb{Z}_{q}^{n \times m}$ find a vector $\mathbf{v} \in \mathbb{Z}^{m} \backslash\{0\}$ such that $\mathbf{A v}=0$ and $\|\mathbf{v}\| \leq \beta$.

In order for the above problem to not be vacuously hard, we need to have $\beta \geq \sqrt{m} q^{n / m}$ in order for there to exist a solution $\mathbf{v}$. The signature scheme that we construct in Section 4 is based on the presumed hardness of the above 
problem. In Section 5, we construct a more efficient signature scheme based on the hardness of SIS variants defined below.

Definition 2.2 ( $\mathrm{SIS}_{q, n, m, d}$ distribution). Choose a random matrix $\mathbf{A} \stackrel{\$}{\leftarrow} \mathbb{Z}_{q}^{n \times m}$ and a vector $\mathbf{s} \stackrel{\$}{\leftarrow}\{-d, \ldots, 0, \ldots, d\}^{m}$ and output $(\mathbf{A}, \mathbf{A s})$.

Definition 2.3 (SIS $_{q, n, m, d}$ search problem). Given a pair (A,t) from the SIS $_{q, n, m, d}$ distribution, find $a \mathbf{s} \in\{-d, \ldots, 0, \ldots, d\}^{m}$ such that $\mathbf{A s}=\mathbf{t}$.

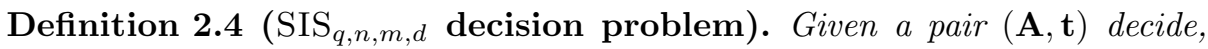
with non-negligible advantage, whether it came from the $\mathrm{SIS}_{q, n, m, d}$ distribution or whether it was generated uniformly at random from $\mathbb{Z}_{q}^{n \times m} \times \mathbb{Z}_{q}^{n}$.

Depending on the relationship between its parameters, the $\mathrm{SIS}_{q, n, m, d}$ search (and decision) problem has somewhat different characteristics. If, for example, we have $d \ll q^{n / m}$, then with very high probability there is only one vector s whose coefficients have absolute value at most $d$ such that $\mathbf{A s}=\mathbf{t}$, and such instances of the $\mathrm{SIS}_{q, n, m, d}$ problem are said to be low-density instances (borrowing from terminology used to describe instances of the random subset sum problem). On the other hand, if $d \gg q^{n / m}$ then the $\operatorname{SIS}_{q, n, m, d}$ distribution is actually statistically close to uniform over $\mathbb{Z}_{q}^{n \times m} \times \mathbb{Z}_{q}^{n}$ (by the leftover hash lemma) and there are many possible solutions $\mathbf{s}$ for which $\mathbf{A s}=\mathbf{t}$. These instances are traditionally called high-density instances. The hardness of the SIS problem is discussed in the full version of this work, but we will just mention that the hardest instances are those in which $d \approx q^{n / m}$.

Notice that if $m \geq 2 n$, then the matrix $\mathbf{A} \stackrel{\$}{\leftarrow} \mathbb{Z}_{q}^{n \times m}$ will, with high probability, contain $n$ columns that are linearly independent over $\mathbb{Z}_{q}$ (when $m \geq 2 n$ and $q$ is a prime of size at least $2 m$, this will be true with probability $\left.e^{-\Omega(n)}\right)$. Without loss of generality, assume that the last $n$ columns of $\mathbf{A}$ are linearly independent, and so $\mathbf{A}=\left[\mathbf{A}_{1} \| \mathbf{A}_{2}\right]$ where $\mathbf{A}_{2}$ is an $n \times n$ invertible matrix. If we consider the matrix $\mathbf{A}^{\prime}=\mathbf{A}_{2}^{-1} \mathbf{A}=\left[\mathbf{A}_{2}^{-1} \mathbf{A}_{1}|| \mathbf{I}\right]$, where $\mathbf{I}$ is an $n \times n$ identity matrix, then we have $\mathbf{A} \mathbf{v}=0$ iff $\mathbf{A}^{\prime} \mathbf{v}=0$, and so the $\ell_{2}-S_{1} S_{q, n, m}$ problem is equally hard if the last $n$ columns of the matrix $\mathbf{A}$ form the identity matrix. Similarly, given an instance $(\mathbf{A}, \mathbf{t})$ of the $\operatorname{SIS}_{q, n, m, d}$ problem, we can change it to $\left(\mathbf{A}_{2}^{-1} \mathbf{A}, \mathbf{A}_{2}^{-1} \mathbf{t}\right)$, and a solution for one will be exactly the same as the solution for the other. Therefore throughout this paper we will assume, without loss of generality, that the matrix $\mathbf{A} \in \mathbb{Z}_{q}^{n \times m}$ is of the form $\mathbf{A}=[\overline{\mathbf{A}}|| \mathbf{I}]$, where $\overline{\mathbf{A}}$ is uniformly generated in $\mathbb{Z}_{q}^{n \times(m-n)}$. For reasons related to lattices, when $\mathbf{A}$ is in this form, we will refer to it as being in Hermite Normal Form [32.

\subsection{Relations between the SIS Variants}

We now state some results about the relationship between the SIS variants defined above. The first relationship is an adaptation of a classic theorem of Impagliazzo and Naor [22], who showed that the decisional version of the random subset sum problem is as hard as the search version. This theorem has been recently generalized by Micciancio and Mol [29]. 
Theorem 2.5. 2229] If $d$ is polynomial in $n$, then there is a polynomial-time reduction from the $\mathrm{SIS}_{q, n, m, d}$ search problem to the $\mathrm{SIS}_{q, n, m, d}$ decision problem.

The next lemma shows that the decision $\mathrm{SIS}_{q, n, m, d}$ problem gets harder when the value of $d$ increases. This is a rather intuitive result since the decision $\operatorname{SIS}_{q, n, m, d}$ problem becomes vacuously hard when $d \gg q^{n / m}$ since the $\mathrm{SIS}_{q, n, m, d}$ distribution will be statistically close to uniform.

Lemma 2.6. For any non-negative integer $\alpha$ such that $\operatorname{gcd}(2 \alpha+1, q)=1$, there is a polynomial-time reduction from the $\mathrm{SIS}_{q, n, m, d}$ decision problem to the $\mathrm{SIS}_{q, n, m,(2 \alpha+1) d+\alpha}$ decision problem.

The final lemma that we prove shows that if $m=2 n$ and one can solve the can solve $\ell_{2}-\mathrm{SIS}_{q, n, m, \beta}$ problem for a small-enough $\beta$, then one can solve the decision $\mathrm{SIS}_{q, n, m, d}$ problem. This result is essentially folklore (see [32]), but we state it here for completeness.

Lemma 2.7. If $m=2 n$ and $4 d \beta \leq q$, then there is a polynomial-time reduction from solving the $\mathrm{SIS}_{q, n, m, d}$ decision problem to the $\ell_{2}-\mathrm{SIS}_{q, n, m, \beta}$ problem.

\section{Rejection Sampling and the Normal Distribution}

Definition 3.1. The continuous Normal distribution over $\mathbb{R}^{m}$ centered at $\mathbf{v}$ with standard deviation $\sigma$ is defined by the function $\rho_{\mathbf{v}, \sigma}^{m}(\mathbf{x})=\left(\frac{1}{\sqrt{2 \pi \sigma^{2}}}\right)^{m} e^{\frac{-\|\mathbf{x}-\mathbf{v}\|^{2}}{2 \sigma^{2}}}$

When $\mathbf{v}=0$, we will just write $\rho_{\sigma}^{m}(\mathbf{x})$. We will define the discrete Normal distribution over $\mathbb{Z}^{m}$ as follows:

Definition 3.2. The discrete Normal distribution over $\mathbb{Z}^{m}$ centered at some $\mathbf{v} \in \mathbb{Z}^{m}$ with standard deviation $\sigma$ is defined as $D_{\mathbf{v}, \sigma}^{m}(\mathbf{x})=\rho_{\mathbf{v}, \sigma}^{m}(\mathbf{x}) / \rho_{\sigma}^{m}\left(\mathbb{Z}^{m}\right)$.

In the above definition, the quantity $\rho_{\sigma}^{m}\left(\mathbb{Z}^{m}\right)=\sum_{\mathbf{z} \in \mathbb{Z}^{m}} \rho_{\sigma}^{m}(\mathbf{z})$ is just a scaling quantity needed to make the function into a probability distribution. Also note that for all $\mathbf{v} \in \mathbb{Z}^{m}, \rho_{\mathbf{v}, \sigma}^{m}\left(\mathbb{Z}^{m}\right)=\rho_{\sigma}^{m}\left(\mathbb{Z}^{m}\right)$, thus the scaling factor is the same for all $\mathbf{v}$.

The below lemma collects some basic facts about the discrete Normal distribution over $\mathbb{Z}^{m}$. These results are special cases of more general results about the discrete Normal distribution over arbitrary lattices from [6]3137.

\section{Lemma 3.3}

1. $\operatorname{Pr}\left[|z|>\omega(\sigma \sqrt{\log m}) ; z \stackrel{\$}{\leftarrow} D_{\sigma}^{1}\right]=2^{-\omega(\log m)}$, and more specifically, $\operatorname{Pr}\left[|z|>12 \sigma ; z \stackrel{\$}{\leftarrow} D_{\sigma}^{1}\right]<2^{-100}$.

2. For any $\mathbf{z} \in \mathbb{Z}^{m}$, and $\sigma \geq \sqrt{\log 3 m}, D_{\sigma}^{m}(\mathbf{z}) \leq 2^{-m+1}$.

3. $\operatorname{Pr}\left[\|\mathbf{z}\|>2 \sigma \sqrt{m} ; \mathbf{z} \stackrel{\$}{\leftarrow} D_{\sigma}^{m}\right]<2^{-m}$.

We now state the main theorem of this section whose proof is given in the full version of this paper. 
Signing Key: $\mathbf{S} \stackrel{\$}{\leftarrow}\{-d, \ldots, 0, \ldots, d\}^{m \times k}$

Verification Key: $\mathbf{A} \stackrel{\$}{\leftarrow} \mathbb{Z}_{q}^{n \times m}, \mathbf{T} \leftarrow \mathbf{A S}$

Random Oracle: $\mathrm{H}:\{0,1\}^{*} \rightarrow\left\{\mathbf{v}: \mathbf{v} \in\{-1,0,1\}^{k},\|\mathbf{v}\|_{1} \leq \kappa\right\}$

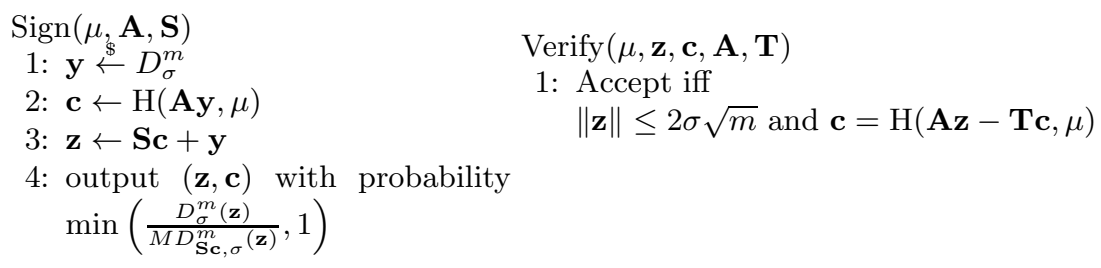

Fig. 1. Signature Scheme

Theorem 3.4. Let $V$ be a subset of $\mathbb{Z}^{m}$ in which all elements have norms less than $T, \sigma$ be some element in $\mathbb{R}$ such that $\sigma=\omega(T \sqrt{\log m})$, and $h: V \rightarrow \mathbb{R}$ be a probability distribution. Then there exists a constant $M=O(1)$ such that the distribution of the following algorithm $\mathcal{A}$ :

1: $\mathbf{v} \stackrel{\$}{\leftarrow} h$

2: $\mathbf{z} \stackrel{\$}{\leftarrow} D_{\mathbf{v}, \sigma}^{m}$

3: output $(\mathbf{z}, \mathbf{v})$ with probability $\min \left(\frac{D_{\sigma}^{m}(\mathbf{z})}{M D_{\mathbf{v}, \sigma}^{m}(\mathbf{z})}, 1\right)$

is within statistical distance $\frac{2^{-\omega(\log m)}}{M}$ of the distribution of the following algorithm $\mathcal{F}$ :

1: $\mathbf{v} \stackrel{\$}{\leftarrow} h$

2: $\mathbf{z} \stackrel{\$}{\leftarrow} D_{\sigma}^{m}$

3: output $(\mathbf{z}, \mathbf{v})$ with probability $1 / M$

Moreover, the probability that $\mathcal{A}$ outputs something is at least $\frac{1-2^{-\omega(\log m)}}{M}$.

More concretely, if $\sigma=\alpha T$ for any positive $\alpha$, then $M=e^{12 / \alpha+1 /\left(2 \alpha^{2}\right)}$, the output of algorithm $\mathcal{A}$ is within statistical distance $\frac{2^{-100}}{M}$ of the output of $\mathcal{F}$, and the probability that $\mathcal{A}$ outputs something is at least $\frac{1-2^{-100}}{M}$.

\section{Signature Scheme Based on SIS}

In this section we present our main theoretical result - a signature scheme based, in the random oracle model, on the average-case hardness of the $\ell_{2}$-SIS $\mathrm{SI}_{q, m, \beta}$ problem for $\beta=\tilde{O}(n)$. The scheme is presented in Figure 1 and the definition of its parameters and some sample instantiations are in Figure 2. We will now explain the workings of the scheme and sketch the intuition for its security.

The secret key is an $m \times k$ matrix $\mathbf{S}$ of random integers of absolute value at most $d$, and the public key consists of a random matrix $\mathbf{A} \in \mathbb{Z}_{q}^{n \times m}$ and another matrix $\mathbf{T} \in \mathbb{Z}_{q}^{m \times k}$ which is equal to AS. For concreteness, we will consider distributions to be statistically close if they are $\approx 2^{-100}$ apart, and we will also 


\begin{tabular}{|c||c|c|c|c|c|}
\hline & I & II & III & IV & V \\
\hline \hline $\mathrm{n}$ & 512 & 512 & 512 & 512 & 512 \\
\hline $\mathrm{q}$ & $2^{27}$ & $2^{25}$ & $2^{33}$ & $2^{24}$ & $2^{33}$ \\
\hline $\mathrm{d}$ & 1 & 1 & 31 & 1 & 31 \\
\hline $\mathrm{k}$ & 80 & 512 & 512 & 512 & 512 \\
\hline$m \approx 64+n \cdot \log q / \log (2 d+1)$ & 8786 & 8139 & 3253 & - & - \\
\hline$m=2 n(\mathrm{used}$ in Section 5 ) & - & - & - & 1024 & 1024 \\
\hline$\kappa$ s.t. $2^{\kappa} \cdot\left(\begin{array}{l}n \\
\kappa\end{array}\right) \geq 2^{100}$ & 28 & 14 & 14 & 14 & 14 \\
\hline$\sigma \approx 12 \cdot d \cdot \kappa \cdot \sqrt{m}$ & 31495 & 15157 & 300926 & 5376 & 166656 \\
\hline$M \approx \exp \left(12 d \kappa \sqrt{m} / \sigma+(d \kappa \sqrt{m} / 2 \sigma)^{2}\right)$ & 2.72 & 2.72 & 2.72 & 2.72 & 2.72 \\
\hline signature size $(\mathrm{bits}) \approx m \log (12 \sigma)$ & 163000 & 142300 & 73000 & 16500 & 20500 \\
\hline secret key size $(\mathrm{bits}) \approx m \cdot k \cdot \log (2 d+1)$ & $2^{20}$ & $2^{22.5}$ & $2^{23}$ & $2^{19.5}$ & $2^{21.5}$ \\
\hline public key size $(\mathrm{bits}) \approx n \cdot k \cdot \log q$ & $2^{20}$ & $2^{22.5}$ & $2^{23}$ & $2^{22.5}$ & $2^{23}$ \\
\hline
\end{tabular}

Fig. 2. Signature Scheme Parameters. The parameters in columns I, II, and III are based on the hardness of the $\ell_{2}$-SIS ${ }_{q, n, m, \beta}$ problem where for the $\beta$ in Theorem 4.1 Columns IV and V are based on the hardness of the $\mathrm{SIS}_{q, n, m, d}$ search problem (see Section 5). Furthermore, the parameters in column V are also compatible with the LWE assumption (see Section [5.1. The security level for all the instantiations is for $\delta \approx 1.007$ (see the full version of this paper). For the ring-based instantiations, described in the full version, the key sizes are smaller by a factor of $k$.

want $\approx 100$ bits of security from our cryptographic hash function $\mathrm{H}$, and so we will assume that the output of $\mathrm{H}$ is 100 bits 4

To sign a message $\mu$, the signer first picks an m-dimensional vector $\mathbf{y}$ from the distribution $D_{\sigma}^{m}$, for some standard deviation $\sigma$, then computes $\mathbf{c}=\mathrm{H}(\mathbf{A y}, \mu)$, and finally computes $\mathbf{z}=\mathbf{S c}+\mathbf{y}$ (there is no reduction modulo $q$ in this step!). The potential signature which he outputs is $(\mathbf{z}, \mathbf{c})$, but he only outputs it with probability $\min \left(\frac{D_{\sigma}^{m}(\mathbf{z})}{M D_{\mathbf{S c}, \sigma}^{m}(\mathbf{z})}, 1\right)$. If nothing was output, the signer runs the signing algorithm again until some signature is outputted.

The main idea behind this structure of the signing algorithm is to make the distribution of the signature $(\mathbf{z}, \mathbf{c})$ independent of the secret key $\mathbf{S}$. The target distribution for the $\mathbf{z}$ 's that we will be aiming for is $D_{\sigma}^{m}$, but the elements $\mathbf{z}$ in the signature scheme come from the distribution $D_{\mathbf{v}, \sigma}^{m}$, where $\mathbf{v}=\mathbf{S c}$. This is where we will apply the rejection sampling theorem, Theorem 3.4 from Section 3 to show that for an appropriately-chosen value of $M$ and $\sigma$, the signature algorithm will output something with probability approximately $1 / M$ and the statistical distance between its output is statistically close to the distribution in which $\mathbf{z}$ is chosen from $D_{\sigma}^{m}$.

\footnotetext{
${ }^{4}$ It is generally considered folklore that for obtaining signatures with $\lambda$ bits of security using the Fiat-Shamir transform, one only needs random oracles that output $\lambda$ bits (i.e. collision-resistance is not a requirement). While finding collisions in the random oracle does allow the valid signer to produce two distinct messages that have the same signature, this does not constitute a break.
} 
Once we decoupled the distribution of the signature from the distribution of the secret key, we can use a forger who successfully breaks the signature to solve the $\ell_{2}-\mathrm{SIS}_{q, n, m, \beta}$ problem for $\beta \approx \tilde{O}(\|\mathbf{z}\|)$. The idea is that given an $\mathbf{A}$, one can create a secret key $\mathbf{S}$ and publish the public key $(\mathbf{A}, \mathbf{A S})$. Then one can reply to signing queries of the forger by either using the key $\mathbf{S}$, or simply by producing signatures by generating $\mathbf{z}$ from the distribution $D_{\sigma}^{m}$ and programming the random oracle accordingly. In our proof (Lemma 4.4), we choose the latter approach because in Section 5 we will not know a valid secret key, but we would like to be able to still use the the same lemma there. Once we have a way to reply to signing queries, we use the forking lemma [387] to use the forger's valid signatures to recover a short vector $\mathbf{v}$ such that $\mathbf{A v}=0$. One important caveat is that to prove that $\mathbf{v} \neq 0$, there needs to be a second (unknown to us) valid secret key $\mathbf{S}^{\prime}$ such that $\mathbf{A S}=\mathbf{A} \mathbf{S}^{\prime}$, and the forger cannot know which secret key we know. To satisfy the existence of another secret key requires a particular relationship between $n, m$, and $q$ (Lemma 4.2), and the indistinguishability of $\mathbf{S}$ and $\mathbf{S}^{\prime}$ is clearly satisfied because the distribution of the signature is independent of the secret key.

We now discuss the verification procedure. Since we tailored $\mathbf{z}$ to be distributed according to $D_{\sigma}^{m}$, by Lemma 3.3, we know that with probability at least $1-2^{-m}$, we have $\|\mathbf{z}\|<2 \sigma \sqrt{m}$. And since $\mathbf{A y}=\mathbf{A} \mathbf{z}-\mathbf{T c}$, the second part of the verification will accept a valid signature.

Theorem 4.1. If there is a polynomial-time forger, who makes at most $s$ queries to the signing oracle and $h$ queries to the random oracle $H$, who breaks the signature in Figure 1 (with the relationship between the parameters as in Figure 2) with probability $\delta$, then there is a polynomial-time algorithm who can solve the $\ell_{2}-\mathrm{SIS}_{q, n, m, \beta}$ problem for $\beta=(4 \sigma+2 d \kappa) \sqrt{m}=\tilde{O}(d n)$ with probability $\approx \frac{\delta^{2}}{2(h+s)}$. Moreover, the signing algorithm produces a signature with probability $\approx 1 / M$ and the verifying algorithm accepts a signature produced by an honest signer with probability at least $1-2^{-m}$.

Proof. The theorem is proved in a sequence of two Lemmas. In Lemma 4.3 we show that our signing algorithm can be replaced by the one in Hybrid 2 of Figure 3, and the statistical distance between the two outputs will be at most $\epsilon=s(h+s) \cdot 2^{-n+1}+s \cdot \frac{2^{-100}}{M}$. Since Hybrid 2 produces an output with probability exactly $1 / M$, the signing algorithm produces an output with probability at least $(1-\epsilon) / M$. Then in Lemma 4.4, we show that if a forger can produce a forgery with probability $\delta$ when when the signing algorithm is replaced by one in Hybrid 2 , then we can use him to recover a vector $\mathbf{v}$ such that $\|\mathbf{v}\| \leq(4 \sigma+2 d \kappa) \sqrt{m}$ and $\mathbf{A v}=0$ with probability at least $\left(\frac{1}{2}-2^{-100}\right)\left(\delta-2^{-100}\right)\left(\frac{\delta-2^{-100}}{h+s}-2^{-100}\right) \approx$ $\frac{\delta^{2}}{2(h+s)}$.

Lemma 4.2. For any $\mathbf{A} \in \mathbb{Z}_{q}^{n \times m}$ where $m>64+n \cdot \log q / \log (2 d+1)$, for randomly chosen $\mathbf{S} \stackrel{\$}{\leftarrow}\{-d, \ldots, 0, \ldots, d\}^{m}$, with probability $1-2^{-100}$, there exists another $\mathbf{s}^{\prime} \in\{-d, \ldots, 0, \ldots, d\}^{m}$ such that $\mathbf{A} \mathbf{s}=\mathbf{A} \mathbf{s}^{\prime}$. 
$\underline{\text { Hybrid } 1}$

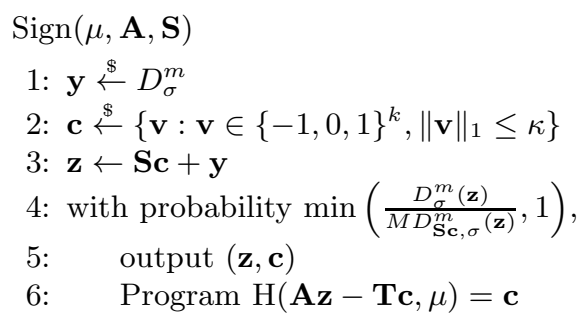

Hybrid 2

$$
\begin{aligned}
& \operatorname{Sign}(\mu, \mathbf{A}, \mathbf{S}) \\
& \text { 1: } \mathbf{c} \stackrel{\$}{\leftarrow}\left\{\mathbf{v}: \mathbf{v} \in\{-1,0,1\}^{k},\|\mathbf{v}\|_{1} \leq \kappa\right\} \\
& \text { 2: } \mathbf{z} \stackrel{\$}{\leftarrow} D_{\sigma}^{m} \\
& \text { 3: } \text { with probability } 1 / M, \\
& \text { 4: } \quad \text { output }(\mathbf{z}, \mathbf{c}) \\
& \text { 5: } \quad \text { Program } \mathrm{H}(\mathbf{A z}-\mathbf{T} \mathbf{c}, \mu)=\mathbf{c}
\end{aligned}
$$

Fig. 3. Signing Hybrids

Lemma 4.3. Let $\mathcal{D}$ be a distinguisher who can query the random oracle $H$ and either the actual signing algorithm in Figure 1 or Hybrid 2 in Figure [3. If he makes $h$ queries to $H$ and $s$ queries to the signing algorithm that he has access to, then for all but a $e^{-\Omega(n)}$ fraction of all possible matrices $\mathbf{A}$, his advantage of distinguishing the actual signing algorithm from the one in Hybrid 2 is at most $s(h+s) \cdot 2^{-n+1}+s \cdot \frac{2^{-\omega(\log m)}}{M}$, or more concretely, $s(h+s) \cdot 2^{-n+1}+s \cdot \frac{2^{-100}}{M}$.

Proof. We first show that the distinguisher $\mathcal{D}$ has advantage of at most $s(h+$ $s) 2^{-n+1}$ of distinguishing between the real signature scheme and Hybrid 1 . The only difference between the actual signing algorithm and the algorithm in Hybrid 1 is that in Hybrid 1, the output of the random oracle $\mathrm{H}$ is chosen at random from $\left\{\mathbf{v}: \mathbf{v} \in\{-1,0,1\}^{k},\|\mathbf{v}\|_{1} \leq \kappa\right\}$ and then programmed as the answer to $\mathrm{H}(\mathbf{A z}-\mathbf{T} \mathbf{c}, \mu)=\mathrm{H}(\mathbf{A y}, \mu)$ without checking whether the value for $(\mathbf{A y}, \mu)$ was already set. Since $\mathcal{D}$ calls $H h$ times, and the signing algorithm $s$ times, at most $s+h$ values of $(\mathbf{A y}, \mu)$ will ever be set. We now show that each time the Hybrid 1 procedure is called, the probability of generating a $\mathbf{y}$ such that $\mathbf{A y}$ is equal to one of the previous values that was queried is at most $2^{-n+1}$. With probability at least $1-e^{-\Omega(n)}$, the matrix $\mathbf{A}$ can be written in "Hermite Normal Form" (see Section 2] as $\mathbf{A}=[\overline{\mathbf{A}} \| \mathbf{I}]$. Then, for any $\mathbf{t} \in \mathbb{Z}_{q}^{n}$,

$$
\begin{aligned}
\operatorname{Pr}\left[\mathbf{A y}=\mathbf{t} ; \mathbf{y} \stackrel{\$}{\leftarrow} D_{\sigma}^{m}\right] & =\operatorname{Pr}\left[\mathbf{y}_{1}=\left(\mathbf{t}-\overline{\mathbf{A}} \mathbf{y}_{0}\right) ; \mathbf{y} \stackrel{\$}{\leftarrow} D_{\sigma}^{m}\right] \\
& \leq \max _{\mathbf{t}^{\prime} \in \mathbb{Z}_{q}^{n}} \operatorname{Pr}\left[\mathbf{y}_{1}=\mathbf{t}^{\prime} ; \mathbf{y}_{1} \stackrel{\$}{\leftarrow} D_{\sigma}^{n}\right] \leq 2^{-n+1},
\end{aligned}
$$

where the last inequality follows from Lemma 3.3. Thus if Hybrid 1 is accessed $s$ times, and the probability of getting a collision each time is at most $(s+h) 2^{-n+1}$, the probability that a collision occurs after $s$ queries is at most $s(s+h) 2^{-n+1}$.

We next show that the statistical distance between the outputs of Hybrid 1 and Hybrid 2 is at most $\frac{2^{-\omega(\log m)}}{M}$. The proof of this fact is almost a direct consequence of Theorem 3.4. Notice that if both Hybrids simply outputted $(\mathbf{z}, \mathbf{v}=\mathbf{S c})$ with probability $\min \left(\frac{D_{\sigma}^{m}(\mathbf{z})}{M D_{\mathbf{S c}, \sigma}^{m}(\mathbf{z})}, 1\right)$ for Hybrid 1 and probability $1 / M$ for Hybrid 2, then Hybrid 1 exactly plays the role of the algorithm $\mathcal{A}$ in Theorem 3.4 and Hybrid 2 corresponds to $\mathcal{F}$ (where the maximum $T$ in Theorem 3.4 corresponds to $d \kappa \sqrt{m}$ ). 
But instead of outputting $\mathbf{v}=\mathbf{S c}$, the Hybrids output just $\mathbf{c}$. But this does not increase the statistical distance because given $\mathbf{v}$, one can generate $\mathbf{c}$ by picking a random element $\mathbf{c} \in\left\{\mathbf{w}: \mathbf{w} \in\{-1,0,1\}^{k},\|\mathbf{w}\|_{1} \leq \kappa\right\}$ such that $\mathbf{S c}=\mathbf{v}$ (for our choice of parameters in this paper, there will actually be only one possible $\mathbf{c}$, with very high probability), and this will have the exact same distribution as the $\mathbf{c}$ in both Hybrids. And finally, since the signing oracle is called $s$ times, the statistical distance is no more than $s \cdot \frac{2^{-\omega(\log m)}}{M}$, or more concretely, $s \cdot \frac{2^{-100}}{M}$, (since we set $\sigma=12 T)$, and we obtain the claim in the lemma.

Lemma 4.4. Suppose there exists a polynomial-time forger $\mathcal{F}$ who makes at most $h$ queries to the signer in Hybrid 2, s queries to the random oracle $H$, and succeeds in forging with probability $\delta$. Then there exists an algorithm of the same time-complexity as $\mathcal{F}$ that for a given $\mathbf{A} \stackrel{\$}{\leftarrow} \mathbb{Z}_{q}^{n \times m}$ finds a non-zero $\mathbf{v} \in \mathbb{Z}^{m}$ such that $\|\mathbf{v}\| \leq(4 \sigma+2 d \kappa) \sqrt{m}$ and $\mathbf{A v}=0$ with probability at least

$$
\left(\frac{1}{2}-2^{-100}\right)\left(\delta-2^{-100}\right)\left(\frac{\delta-2^{-100}}{h+s}-2^{-100}\right) .
$$

\subsection{Setting the Parameters}

In Figure2, we set some sample parameters to demonstrate the influence of their interplay on the sizes of the signature length and the key size. The secret key is an $m \times k$ matrix with coefficients having absolute value at most $d$, and so it can be represented by $m k \log (2 d+1)$ bits. The public key $\mathbf{A}, \mathbf{T}$ can be spit into two parts - the matrix $\mathbf{A}$ can be shared by all users (and so can be considered as part of the function), whereas the matrix $\mathbf{T}$ is individual. The part of the public key that is individual for each user requires $n k \log q$ bits of storage. The signature size is dominated by the vector $\mathbf{z}$, since $\mathbf{c}$ is just a small bit-string that is the output of the cryptographic hash function $\mathrm{H}$. By design, the vector $\mathbf{z}$ is distributed according to $D_{\sigma}^{m}$, and by Lemma 3.3, we know that with probability at least $1-2^{-100}$, each coefficient of $\mathbf{z}$ is of length at most $12 \sigma$. Thus $\mathbf{z}$ can be represented by $m \log (12 \sigma)$ bits.

For security, we use the analysis of [1332] (also discussed in the full version of this paper), where it is shown that the smallest vector $\mathbf{v}$ such that $\mathbf{A v}=0$ can be produced has length $\min \left(q, 2^{2 \sqrt{n \log q \log \delta}}\right)$. We would like this vector $\mathbf{v}$ to have a larger size than the vector that can be extracted from the successful forger, which is given in Lemma 4.4. There are some trade-offs between the sizes of signatures and keys that can be achieved for the same security level. For example, if we change the value of $k$ from 80 in column I to 512 in column II, it has the effect of making the keys larger by a factor of around 6 , and at the same time reducing the signature size by a little over $10 \%$. Another interesting trade-off is achieved by raising the value of $d$ as in column III. Notice that what most affects the length of the signature size is the parameter $m$. By raising the value of $d$ and $q$, we can lower $m$, and can reduce the signature size by almost $50 \%$ at the expense of slightly increasing the key sizes. 


\section{Signatures Based on Low-Density SIS and LWE}

From the sample instantiations in the previous section, we saw that $m$ is the one parameter that most affects the signature size. In this section we explore the results of breaking the requirement that $m \approx 64+n \cdot \log q / \log (2 d+1)$ (which is required for Lemma 4.2) and show that this still gives us a provably-secure signature scheme (based on the low-density SIS $_{q, n, m, d}$ problem), but with much smaller signature and key sizes. Let us consider, for example, taking instantiation III in Figure 2 and lowering the value of $d$ from 31 to, say, 1, without changing the value of $m$. The potential advantage of this modification is that the value of $\sigma$ goes down by a factor of $d$, which has the effect of making the signature vector $\mathbf{z}$ smaller (by a factor $d$ ), which in turn makes it harder for the adversary to produce a forgery, since he now needs to find a vector that is $d$ times smaller than before. This in turn allow us to lower other parameters, such as $q$ and $m$, which leads to a "virtuous cycle" of reducing the length of the signature.

We now look at what happens to the security proofs if we proceed as described above. The main problem is that Lemma 4.2 is no longer true since for every $\mathbf{T}$, there will now be, with extremely high probability, only one $\mathbf{S}$ for which $\mathbf{A S}=\mathbf{T}$. The fact that there were multiple $\mathbf{S}$ 's was crucially used at the end of Lemma 4.4 to argue that a successful forger can be used to extract a small vector $\mathbf{v}$ such that $\mathbf{A v}=0$. On the other hand, the proof of Lemma 4.3 is not affected by the relationship between $d$ and $m$, and so the real signature scheme is still indistinguishable from one that uses Hybrid 2 as its signing algorithm. And since Hybrid 2 does not use the secret key to produce signatures, for a given $\mathbf{A}$, we can use the secret key $\mathbf{S}$ with small coefficients in the actual signature, but use an $\mathbf{S}^{\prime}$ with large coefficients (so that there exists an $\mathbf{S}^{\prime \prime}$ such that $\mathbf{A} \mathbf{S}^{\prime}=\mathbf{A} \mathbf{S}^{\prime \prime}$ ) in the proof (see Figure 4). If the distribution of the verification key $(\mathbf{A}, \mathbf{A S})$ is computationally indistinguishable from that of $\left(\mathbf{A}, \mathbf{A} \mathbf{S}^{\prime}\right)$ (and it is, based on the hardness of the low-density $\mathrm{SIS}_{q, n, m, d}$ problem from Definition 2.4), the distinguisher will not be able to tell that he is given an invalid key pair. And since we never use the secret key to provide signatures to the forger in Lemma 4.4 the forger should act in the same way, and we will be able to find a non-zero $\mathbf{v}$ such that $\mathbf{A v}=0$.

Using the above framework, we can obtain a signature scheme that is based on the hardness of two problems (i.e. both problems need to be hard for our scheme to be secure): the $\operatorname{SIS}_{q, n, m, d}$ decisional (and by Theorem[2.5, also computational) problem and the $\ell_{2}$-SIS I $, n, m, \beta$ problem with $\beta=\left(4 \sigma+2 d^{\prime} \kappa\right) \sqrt{m}$. Thus the optimal parameter settings will be where the two problems are equally hard. Furthermore, if we set $m=2 n$ and have $4 d \beta \leq q$, then the $\operatorname{SIS}_{q, n, m, d}$ problem reduces to the $\ell_{2}$-SIS $q, n, m, \beta$ one (Lemma 2.7), and we end up with just one simple computational hardness assumption - $\mathrm{SIS}_{q, n, m, d}$. We formalize the above intuition in two lemmas analogous to Lemmas 4.3 and 4.4 from Section 4

Lemma 5.1. Let $\mathcal{D}$ be a distinguisher who can query the random oracle $H$ and either the actual key-generation/signing algorithms in Figure 1 or those in 
Hybrid 2

Signing Key: $\mathbf{S} \stackrel{\$}{\leftarrow}\{-d, \ldots, 0, \ldots, d\}^{m \times k}$ Verification Key: $\mathbf{A} \stackrel{\$}{\leftarrow} \mathbb{Z}_{q}^{n \times m}, \mathbf{T} \leftarrow \mathbf{A S}$
Hybrid 3

Signing Key: $\mathbf{S} \stackrel{\$}{\leftarrow}\left\{-d^{\prime}, \ldots, 0, \ldots, d^{\prime}\right\}^{m \times k}$ Verification Key: $\mathbf{A} \stackrel{\$}{\leftarrow} \mathbb{Z}_{q}^{n \times m}, \mathbf{T} \leftarrow \mathbf{A S}$

$\operatorname{Sign}(\mu, \mathbf{A}, \mathbf{S})$

1: $\mathbf{c} \stackrel{\$}{\leftarrow}\left\{\mathbf{v}: \mathbf{v} \in\{-1,0,1\}^{k},\|\mathbf{v}\|_{1} \leq \kappa\right\}$

2: $\mathbf{z} \stackrel{\$}{\leftarrow} D_{\sigma}^{m}$

3: with probability $1 / M$,

4: $\quad$ output $(\mathbf{z}, \mathbf{c})$

5: $\quad$ Program $\mathrm{H}(\mathbf{A z}-\mathbf{T c}, \mu)=\mathbf{c}$

Fig. 4. Key-Generation and Signing Hybrids: $d^{\prime}$ is set so that $d^{\prime}=(2 \alpha+1) d+\alpha$ for some positive integer $\alpha$ and $m \geq 64+n \cdot \log q / \log \left(2 d^{\prime}+1\right)$

Hybrid 3 in Figure 4 . If he makes $h$ queries to $H$ and $s$ queries to the signing algorithm that he has access to, and can distinguish the real world from Hybrid 3 with advantage $\delta$, then he has advantage $\Omega(\delta / k)-\left(s(h+s) \cdot 2^{-n+1}+s \cdot \frac{2^{-\omega(\log m)}}{M}\right)$ in solving the $\mathrm{SIS}_{q, n, m, d}$ decision problem.

Lemma 5.2. Suppose there exists a polynomial-time forger $\mathcal{F}$ who is given the verification key and access to the signing algorithm from Hybrid 3, and makes at most $h$ queries to the signing algorithm, s queries to the random oracle $H$, and succeeds in forging with probability $\delta$. Then there exists an algorithm of the same time-complexity as $\mathcal{F}$ that for a given $\mathbf{A} \stackrel{\$}{\leftarrow} \mathbb{Z}_{q}^{n \times m}$ finds a $\mathbf{v} \in \mathbb{Z}^{m}$ such that $\|\mathbf{v}\| \leq\left(4 \sigma+2 d^{\prime} \kappa\right) \sqrt{m}$ and $\mathbf{A} \mathbf{v}=0$ with probability at least

$$
\left(\frac{1}{2}-2^{-100}\right)\left(\delta-2^{-100}\right)\left(\frac{\delta-2^{-100}}{h+s}-2^{-100}\right) \text {. }
$$

Proof. The proof is exactly the same as the one of Lemma 4.4, with $d^{\prime}$ playing the role of $d$.

\subsection{The LWE Problem}

In the Learning With Errors (LWE) problem, one is given an oracle that produces ordered pairs of the form $\left(\mathbf{a}_{i}, b_{i}\right) \in \mathbb{Z}_{q}^{n} \times \mathbb{Z}$ where the $\mathbf{a}_{i}$ are uniformly random in $\mathbb{Z}_{q}^{n}$, and $b_{i}=\mathbf{a}_{i} \cdot \mathbf{s}+e_{i}$ where $\mathbf{s}$ is some secret vector in $\mathbb{Z}_{q}^{n}$ and $e_{i}$ is some "error" of small absolute value. Regev [39] showed that there is a quantum reduction from approximating SIVP in all lattices to solving random instances of LWE when the errors $e_{i}$ come from the discrete Normal distribution $D_{\psi}$, and Peikert later showed a classical reduction to LWE from some different lattice problems [35].

An equivalent version of LWE, as shown in [4], is if the secret key is selected from the distribution $D_{\psi}^{n}$ rather than from the uniform distribution. In addition, Regev also showed that the decisional version of the LWE problem, where one is asked to decide whether the ordered pairs $\left(\mathbf{a}_{i}, b_{i}\right)$ come from the uniform distribution or whether they are generated such that $b_{i}=\mathbf{a}_{i} \cdot \mathbf{s}+e_{i}$, is as hard as the search version. 
Using the above definitions, observe that if we have a matrix $\mathbf{A}=[\overline{\mathbf{A}}|| \mathbf{I}] \in$ $\mathbb{Z}_{q}^{n \times 2 n}$, where $\bar{A} \stackrel{\$}{\leftarrow} \mathbb{Z}_{q}^{n \times n}$, then distinguishing pairs (A, As), where each $\mathbf{s} \stackrel{\$}{\leftarrow}$ $D_{\psi}^{2 n}$, from uniformly distributed pairs in $\mathbb{Z}_{q}^{n \times 2 n} \times \mathbb{Z}_{q}^{2 n}$ is exactly the decisional LWE problem. By the hybrid argument, distinguishing (A, AS), where each column of the $k$ columns of $\mathbf{S}$ is distributed according to $D_{\psi}^{2 n}$, from uniformly distributed pairs in $\mathbb{Z}_{q}^{n \times 2 n} \times \mathbb{Z}_{q}^{2 n \times k}$ is also as hard as LWE. Therefore, except for the distribution of the secret key $\mathbf{S}$, the LWE problem is exactly the low-density $\mathrm{SIS}_{q, n, 2 n, d}$ problem, and so we can easily change the scheme in the previous section based on the hardness of low-density SIS to be based on LWE instead.

The most important feature of the secret key $\mathbf{S}$ that is used in the proofs is the norm of each of its columns. If the norm of $\mathbf{s} \stackrel{\$}{\leftarrow} D_{\psi}^{m}$ is approximately the same as that of a vector $\mathbf{s}^{\prime} \stackrel{\$}{\leftarrow}\{-d, \ldots, 0, \ldots, d\}^{m}$, then the security and correctness of the scheme from this section will go through almost entirely unchanged. It can be seen that if $\psi \approx \sqrt{\frac{d \cdot(d+1)}{3}}$, then the length of $\mathbf{s}$ is approximately the same as that of a vector $\mathbf{s}^{\prime}$ ( around $\sqrt{d(d+1) m / 3})$. So a scheme based on LWE where $\psi \approx 18$ would have approximately the same signature size and key lengths as the scheme in column V of Figure 2 where $d=31$.

Notice that the LWE-based scheme in column V produces signatures that are slightly longer than those produced by the scheme in column IV that is based on the $\mathrm{SIS}_{q, n, 2 n, 1}$ problem. At this point, we are not aware of any algorithms that specifically attack $\operatorname{SIS}_{q, n, 2 n, 1}$ which would justify making the signature longer just so that it is based on the hardness of the LWE problem. But in view of the recent algorithm of Arora and Ge [5, which uses algebraic attacks to attack the LWE problem with very small errors, there may be reasons to think that the instantiation in column $\mathrm{V}$ could be more secure because it uses larger coefficients.

Acknowledgements. I am very grateful to Oded Regev for all his great ideas and suggestions that greatly improved this work. I would also like to thank Daniele Micciancio and Chris Peikert for illuminating discussions about the results of 30. And finally, I thank the anonymous Eurocrypt reviewers for their valuable comments and suggestions.

\section{References}

1. Ajtai, M.: Generating Hard Instances of the Short Basis Problem. In: Wiedermann, J., Van Emde Boas, P., Nielsen, M. (eds.) ICALP 1999. LNCS, vol. 1644, pp. 1-9. Springer, Heidelberg (1999)

2. Albrecht, M.R., Farshim, P., Faugère, J.-C., Perret, L.: Polly Cracker, Revisited. In: Lee, D.H., Wang, X. (eds.) ASIACRYPT 2011. LNCS, vol. 7073, pp. 179-196. Springer, Heidelberg (2011)

3. Alwen, J., Peikert, C.: Generating shorter bases for hard random lattices. Theory Comput. Syst. 48(3), 535-553 (2011)

4. Applebaum, B., Cash, D., Peikert, C., Sahai, A.: Fast Cryptographic Primitives and Circular-Secure Encryption Based on Hard Learning Problems. In: Halevi, S. (ed.) CRYPTO 2009. LNCS, vol. 5677, pp. 595-618. Springer, Heidelberg (2009) 
5. Arora, S., Ge, R.: New Algorithms for Learning in Presence of Errors. In: Aceto, L., Henzinger, M., Sgall, J. (eds.) ICALP 2011, Part I. LNCS, vol. 6755, pp. 403-415. Springer, Heidelberg (2011)

6. Banaszczyk, W.: New bounds in some transference theorems in the geometry of numbers. Mathematische Annalen 296, 625-635 (1993)

7. Bellare, M., Neven, G.: Multi-signatures in the plain public-key model and a general forking lemma. In: ACM Conference on Computer and Communications Security, pp. 390-399 (2006)

8. Boyen, X.: Lattice Mixing and Vanishing Trapdoors: A Framework for Fully Secure Short Signatures and More. In: Nguyen, P.Q., Pointcheval, D. (eds.) PKC 2010. LNCS, vol. 6056, pp. 499-517. Springer, Heidelberg (2010)

9. Brakerski, Z., Vaikuntanathan, V.: Efficient fully homomorphic encryption from (standard) LWE. In: FOCS (2011)

10. Brakerski, Z., Vaikuntanathan, V.: Fully Homomorphic Encryption from RingLWE and Security for Key Dependent Messages. In: Rogaway, P. (ed.) CRYPTO 2011. LNCS, vol. 6841, pp. 505-524. Springer, Heidelberg (2011)

11. Cash, D., Hofheinz, D., Kiltz, E., Peikert, C.: Bonsai Trees, or How to Delegate a Lattice Basis. In: Gilbert, H. (ed.) EUROCRYPT 2010. LNCS, vol. 6110, pp. 523-552. Springer, Heidelberg (2010)

12. Fiat, A., Shamir, A.: How to Prove Yourself: Practical Solutions to Identification and Signature Problems. In: Odlyzko, A.M. (ed.) CRYPTO 1986. LNCS, vol. 263, pp. 186-194. Springer, Heidelberg (1987)

13. Gama, N., Nguyen, P.Q.: Predicting Lattice Reduction. In: Smart, N.P. (ed.) EUROCRYPT 2008. LNCS, vol. 4965, pp. 31-51. Springer, Heidelberg (2008)

14. Gentry, C.: Fully homomorphic encryption using ideal lattices. In: STOC, pp. 169-178 (2009)

15. Gentry, C.: Toward Basing Fully Homomorphic Encryption on Worst-Case Hardness. In: Rabin, T. (ed.) CRYPTO 2010. LNCS, vol. 6223, pp. 116-137. Springer, Heidelberg (2010)

16. Gentry, C., Peikert, C., Vaikuntanathan, V.: Trapdoors for hard lattices and new cryptographic constructions. In: STOC, pp. 197-206 (2008)

17. Gentry, C., Szydlo, M.: Cryptanalysis of the Revised NTRU Signature Scheme. In: Knudsen, L.R. (ed.) EUROCRYPT 2002. LNCS, vol. 2332, pp. 299-320. Springer, Heidelberg (2002)

18. Goldreich, O., Goldwasser, S., Halevi, S.: Public-Key Cryptosystems from Lattice Reduction Problems. In: Kaliski Jr., B.S. (ed.) CRYPTO 1997. LNCS, vol. 1294, pp. 112-131. Springer, Heidelberg (1997)

19. Hoffstein, J., Howgrave-Graham, N., Pipher, J., Silverman, J.H., Whyte, W.: NTRUSIGN: Digital Signatures Using the NTRU Lattice. In: Joye, M. (ed.) CT-RSA 2003. LNCS, vol. 2612, pp. 122-140. Springer, Heidelberg (2003)

20. Hoffstein, J., Pipher, J., Silverman, J.H.: NTRU: A Ring-Based Public Key Cryptosystem. In: Buhler, J.P. (ed.) ANTS 1998. LNCS, vol. 1423, pp. 267-288. Springer, Heidelberg (1998)

21. Hoffstein, J., Pipher, J., Silverman, J.H.: NSS: An NTRU Lattice-Based Signature Scheme. In: Pfitzmann, B. (ed.) EUROCRYPT 2001. LNCS, vol. 2045, pp. 211-228. Springer, Heidelberg (2001)

22. Impagliazzo, R., Naor, M.: Efficient cryptographic schemes provably as secure as subset sum. J. Cryptology 9(4), 199-216 (1996)

23. Kawachi, A., Tanaka, K., Xagawa, K.: Concurrently Secure Identification Schemes Based on the Worst-Case Hardness of Lattice Problems. In: Pieprzyk, J. (ed.) ASIACRYPT 2008. LNCS, vol. 5350, pp. 372-389. Springer, Heidelberg (2008) 
24. Lindner, R., Peikert, C.: Better Key Sizes (and Attacks) for LWE-Based Encryption. In: Kiayias, A. (ed.) CT-RSA 2011. LNCS, vol. 6558, pp. 319-339. Springer, Heidelberg (2011)

25. Lyubashevsky, V.: Lattice-Based Identification Schemes Secure Under Active Attacks. In: Cramer, R. (ed.) PKC 2008. LNCS, vol. 4939, pp. 162-179. Springer, Heidelberg (2008)

26. Lyubashevsky, V.: Fiat-Shamir with Aborts: Applications to Lattice and FactoringBased Signatures. In: Matsui, M. (ed.) ASIACRYPT 2009. LNCS, vol. 5912, pp. 598-616. Springer, Heidelberg (2009)

27. Lyubashevsky, V., Micciancio, D.: Asymptotically Efficient Lattice-Based Digital Signatures. In: Canetti, R. (ed.) TCC 2008. LNCS, vol. 4948, pp. 37-54. Springer, Heidelberg (2008)

28. Lyubashevsky, V., Peikert, C., Regev, O.: On Ideal Lattices and Learning with Errors over Rings. In: Gilbert, H. (ed.) EUROCRYPT 2010. LNCS, vol. 6110, pp. 1-23. Springer, Heidelberg (2010)

29. Micciancio, D., Mol, P.: Pseudorandom Knapsacks and the Sample Complexity of LWE Search-to-Decision Reductions. In: Rogaway, P. (ed.) CRYPTO 2011. LNCS, vol. 6841, pp. 465-484. Springer, Heidelberg (2011)

30. Micciancio, D., Peikert, C.: Trapdoors for Lattices: Simpler, Tighter, Faster, Smaller. In: Pointcheval, D., Johansson, T. (eds.) EUROCRYPT 2012. LNCS, vol. 7237, pp. 700-718. Springer, Heidelberg (2012), Preliminary version, http://eprint.iacr.org/2011/501

31. Micciancio, D., Regev, O.: Worst-case to average-case reductions based on gaussian measures. SIAM J. Comput. 37(1), 267-302 (2007)

32. Micciancio, D., Regev, O.: Lattice-based cryptography. In: Bernstein, D.J., Buchmann, J., Dahmen, E. (eds.) Chapter in Post-quantum Cryptography, pp. 147-191. Springer, Heidelberg (2008)

33. Nguyên, P.Q.: Cryptanalysis of the Goldreich-Goldwasser-Halevi Cryptosystem from Crypto'97. In: Wiener, M. (ed.) CRYPTO 1999. LNCS, vol. 1666, pp. 288-304. Springer, Heidelberg (1999)

34. Nguyen, P.Q., Regev, O.: Learning a parallelepiped: Cryptanalysis of GGH and NTRU signatures. J. Cryptology 22(2), 139-160 (2009)

35. Peikert, C.: Public-key cryptosystems from the worst-case shortest vector problem: extended abstract. In: STOC, pp. 333-342 (2009)

36. Peikert, C.: An Efficient and Parallel Gaussian Sampler for Lattices. In: Rabin, T. (ed.) CRYPTO 2010. LNCS, vol. 6223, pp. 80-97. Springer, Heidelberg (2010)

37. Peikert, C., Rosen, A.: Efficient Collision-Resistant Hashing from Worst-Case Assumptions on Cyclic Lattices. In: Halevi, S., Rabin, T. (eds.) TCC 2006. LNCS, vol. 3876, pp. 145-166. Springer, Heidelberg (2006)

38. Pointcheval, D., Stern, J.: Security arguments for digital signatures and blind signatures. J. Cryptology 13(3), 361-396 (2000)

39. Regev, O.: On lattices, learning with errors, random linear codes, and cryptography. J. ACM 56(6) (2009)

40. Rückert, M., Schneider, M.: Estimating the security of lattice-based cryptosystems. Cryptology ePrint Archive, Report 2010/137 (2010), http://eprint.iacr.org/

41. Stehlé, D., Steinfeld, R.: Making NTRU as Secure as Worst-Case Problems over Ideal Lattices. In: Paterson, K.G. (ed.) EUROCRYPT 2011. LNCS, vol. 6632, pp. 27-47. Springer, Heidelberg (2011) 